of cancerous cells that better represent the composition of a tumour in the body than cancer-cell lines, according to Mathew Garnett at the Wellcome Trust Sanger Institute in Hinxton, UK, Hans Clevers at the Hubrecht Institute in Utrecht, the Netherlands, and their colleagues. They built a small bank of 22 tumour organoids using samples from 20 people with colon cancer, and tested the effects of 83 cancer drugs on the cultures. They found correlations between the activity of specific genes and responses to particular drugs.

Some organoids were also uniquely sensitive or insensitive to certain compounds, so the approach might one day be used to tailor treatments for individuals.

Cell 161, 933-945 (2015)

\section{ASTRONOMY}

\section{Quasar quartet in} galactic nursery

Astronomers have discovered a massive cluster of four quasars - a rare find of galaxies just being born.

Quasars are young, bright galaxies powered by supermassive black holes and are hard to find because this youthful period is brief. Using the W. M. Keck Observatory in Hawaii, Joseph Hennawi of the Max Planck Institute for Astronomy in Heidelberg, Germany, and his colleagues found the quasars (pictured, indicated by arrows) at the heart of one of the largest known nebulae - clouds of gas that, if large enough, can give birth to new galaxies. The quasars are illuminating the surrounding gas and

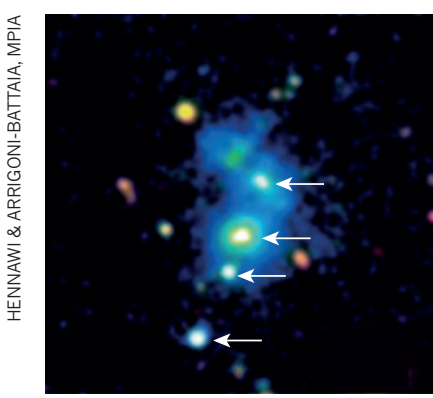

are probably evolving into a massive galaxy cluster.

This rare grouping, together with the size of the nebula, suggests that gas in protogalactic clusters might be cooler and denser than was thought.

Science 348, 779-783 (2015)

\section{CHEMICAL BIOLOG}

\section{Fish makes its own sunscreen}

Zebrafish have the genes needed to synthesize a compound that can provide protection from ultraviolet radiation.

Such chemicals have been found in fish but it was thought that they came from their diet or from microbes that live in or on the animals. Taifo Mahmud at Oregon State University in Corvallis and his colleagues previously analysed fish genomes and discovered genes involved in making these compounds. They then studied the embryos of zebrafish (Danio rerio) and found that their extracts contained the sunscreen compound gadusol. The team inserted the zebrafish genes for gadusol production into yeast (Saccharomyces cerevisiae), which produced milligrams of the compound.

Yeast could be harnessed to make large quantities of the UV protectant, the authors say. eLife 4, e05919 (2015)

\section{ECOLOGY}

\section{Rare bees barely benefit ecosystem}

The sheer number of the most common species in an ecosystem - rather than the level of biodiversity determines how much the system benefits people.

Conservationists have argued that biodiversity supports ecosystem services such as crop pollination. To separate out the effects of species richness from species abundance, Rachael Winfree of Rutgers University in New Brunswick, New Jersey,

SOCIAL SELECTION Ropmatopies

\title{
Fruit-fly paper has 1,000 authors
}

Author lists have grown lengthy in many fields of science, but when a Drosophila genomics paper was published with more than 1,000 authors, it sparked discussion online about the meaning of authorship. The paper, published in the journal G3: Genes Genomes Genetics, names 1,014 authors, with more than 900 undergraduate students among them. Zen Faulkes, an invertebrate neuroethologist at the University of Texas-Pan American in Edinburg, questions on his blog whether every person made enough of a contribution to be credited as an author (see go.nature.com/8rffl7). But the paper's senior author, geneticist Sarah Elgin at Washington University in St. Louis, Missouri, says that large collaborations with correspondingly

SNATURE.COM

For more on popular papers: go.nature.com/gssjdg large author lists have become a fact of life in genomics research. "Putting together the efforts of many people allows you to do good projects," she says. Genes Genomes Genet. 5, 719-740 (2015)
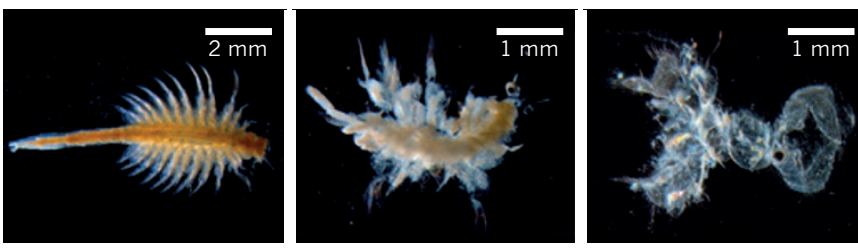

and her colleagues used an equation from evolutionary biology to analyse wild-bee pollination of fruit crops.

The team counted thousands of individual bees from as many as 56 different species in fields of watermelons, blueberries and cranberries, as well as the average number of pollen grains they deposited on flowers. They calculated that pollination was dominated by a few common bee species.

Loss of rare species would not change pollination rates much, but reductions in the number of common bees would make a huge difference, the authors say.

Ecol. Lett. http://doi.org/4m5 (2015)

\section{PALAEONTOLOGY}

\section{Gut microbes give good fossils}

Gut microbes are the main driver of tissue decay when animals die, and were probably important for preserving soft-tissue anatomy in fossil animals.

Philip Donoghue at the University of Bristol, UK, and his colleagues studied the brine shrimp (Artemia salina; pictured left) and monitored its decay (pictured, middle and right) under various conditions. They found that soon after death, the shrimp's gut wall breaks open and bacteria spill out into the body cavity. The bacteria form sticky aggregates, or biofilms, that gradually replace shrimp tissue and contain mineral deposits, as revealed by microscopy. This mineralization is a key step in tissue preservation in fossils.

Evolution of the gut led to an explosion in both animal diversity and the abundance of fossils, the authors say.

Proc. R. Soc. B 282, 20150476 (2015)

\section{$\rightarrow$ NATURE,COM}

For the latest research published by Naturevisit:

www.nature.com/latestresearch 